\title{
Effects of Side Chain Length on Membrane Properties of Copoly- ( $N$-hydroxyalkyl L-glutamine) Hydrogels
}

\author{
Eiji NaKanishi, Yoshihiro Shimizu, Kiyoyuki Ogura, \\ Sadao HibI, and Toshio HAYASHI* \\ Department of Materials Science \& Engineering, Nagoya Institute of Technology, \\ Gokiso-cho, Showa-ku, Nagoya 466, Japan \\ * Research Center for Biomedical Engineering, Kyoto University, \\ 53 Kawahara-cho, Shogoin, Sakyo-ku, Kyoto 606, Japan
}

(Received October 5, 1990)

\begin{abstract}
Random copolymers, poly( $N$-hydroxyethyl L-glutamine-co- $N$-hydroxypentyl L-glutamine) (PHPeEG) and poly $(N$-hydroxypropyl L-glutamine-co- $N$-hydroxypentyl L-glutamine) (PHPePG) as well as the corresponding homopolymers, poly( $N$-hydroxyethyl L-glutamine) (PHEG), poly( $N$-hydroxypropyl L-glutamine) (PHPG), and poly( $N$-hydroxypenthyl L-glutamine) (PHPeG), were prepared by aminolysis in the presence of the crosslinking agent, octamethylenediamine (OMDA), of a membrane of poly( $\gamma$-benzyl-L-glutamate). Membrane properties, such as the degree of swelling $Q_{w}$, conformational property, tensile property, water vapor permeability and enzymatic degradation behavior were studied in the pseudo-extracellular fluid (PECF). $Q_{w}$ and molecular conformation are dependent on the composition of random copolymers. The mechanical strength of PHEG and PHPG hydrogels has been improved largely by introducing HPeG residues. Suitable mechanical property and water vapor permeability were obtained in some copolymers, which were compatible as skin substitutes. When compared at the same $Q_{\mathrm{w}}$, PHPePG copolymers were shown to have higher helix content and mechanical strength, lower water vapor permeability and faster papain digestion than PHPeEG copolymers. This can be explained by differences in hydrophobicity between PHEG and PHPG.
\end{abstract}

KEY WORDS Poly( $N$-hydroxyethyl L-glutamine-co- $N$-hydroxypenthyl Lglutamine) / Poly( $N$-hydroxypropyl L-glutamine-co- $N$-hydroxypentyl Lglutamine) / Hydrogel / Degree of Swelling / Molecular Conformation / Tensile Property / Water Vapor Permeability / Enzymatic Degradation /

Hydrogels are water-swollen hydrophilic materials. Several useful applications of hydrogels require not only suitable degree of swelling, but also favourable elastic and mechanical properties in the swollen state. ${ }^{1,2}$ The molecular design for useful hydrogel which is compatible with high water content and moderate mechanical strength is not so easy. Hydrogels of poly $(\alpha$-aminoacid)s will be useful for medical applications such as temporary artificial skin substitutes, temporary barriers to prevent adhesion, and so on. ${ }^{3}$

In our previous paper, ${ }^{4}$ mechanical properties of random copolymer PHPeEG consisting of PHEG and PHPeG were studied in connection with their molecular conformation. The difference in tensile behavior between PHEG and $\mathrm{PHPeG}$ was interpreted. Tensile property similar to that of human skin was shown in some PHPeEG hydrogels with low Young's modulus and high tensile strength. Polypeptides in PHEG hydrogel exist in random coil conformation while those in PHPG hydrogel exist in interrupted $\alpha$-helix conformation. In this study, random copolymers, PHPeEG and PHPePG, were prepared. The membrane properties of both copolymers, such as the degree of swelling $Q_{\mathrm{w}}$, molecular conformation, 
tensile property, water vapor permeability and enzymatic degradation behavior were investigated in pasudo-extracellular fluid (PECF) ${ }^{5}$ (115 mM NaCl, $30 \mathrm{mM} \mathrm{MaHCO}, 3 \mathrm{mM} \mathrm{KCl}$, and $2 \mathrm{mM} \mathrm{K}_{2} \mathrm{HPO}_{4}$ ) at $\mathrm{pH}=7.4$. The object of this study is to examine membrane properties in connection with molecular conformation, from the viewpoint of possible skin substitutes.

\section{EXPERIMENTAL}

\section{Materials}

The mother polymer, poly $(\gamma$-benzyl Lglutamate) (PBLG) was synthesized by the $N$-carboxyanhydride (NCA) method as described in our previous paper. ${ }^{6}$ Trichloromethyl-chloroformate was supplied by Hodogaya Chemical Co. The molecular weight of PBLG was determined as 335,000 by viscosity measurement ${ }^{7}$ in dichloroacetic acid (DCA). All the solvents used in the synthesis were distilled twice.

Hydrophilic copolymer membranes, PHPeEG and PHPePG, were prepared by the aminolysis $^{8}$ of PBLG membranes cast from chloroform solution. PBLG membranes of ca. $60 \mu \mathrm{m}$ and $1 \mu \mathrm{m}$ in thickness were immersed in a mixture at various compositions of 2-amino1-ethanol (EA) and 5-amino-1-pentanol (PeA) and of 3-amino-1-propanol (PA) and PeA, with $3 \mathrm{~mol} \%$ of the crosslinking agent, 1,8-octamethylenediamine (OMDA), at $58 \pm 0.2^{\circ} \mathrm{C}$. The aminolytic reaction was complete after 7 to 14 days. Debenzylation of $\gamma$-BLG was confirmed by the disappearance of the $a b-$ sorption at $250-260 \mathrm{~nm}$ in UV spectra, and of the absorptions at 700 and $750 \mathrm{~cm}^{-1}$ in IR spectra. The absence of the $\gamma$-benzyl ester group was guaranteed by the disappearance of the absorption due to ester groups at $1730 \mathrm{~cm}^{-1}$ in IR spectra. Compositions of all the copolymers were determined by elementary analysis.

\section{Measurements}

Degree of Swelling $Q_{w}(\%)$ of Hydrogels.
$Q_{\mathrm{w}}(\%)$ was determined by equilibrating the membrane in PECF solution at $37.0^{\circ} \mathrm{C}$. The membrane was then blotted to remove surface PECF and weighed until constant weight was achieved. The membrane was then dried in a vacuum oven. $Q_{\mathrm{w}}(\%)$ was defined as the ratio of the amount of PECF to weight of the dried crosslinked hydrophilic membranes.

Molecular Conformation of Hydrogels. The molecular conformation of linear PHPeEG and PHPePG in PECF solution was examined by circular dichroism (CD) (Jasco J-40 CD/ORD) measurement. The helix content $X_{\mathrm{H}}$ was evaluated from the residue ellipticity $(\theta)$ at $222 \mathrm{~nm}$. The conformations of membranes in xerogel and in hydrogel were examined by IR(Hitachi Model 285) spectra. The Amide V band $^{9}$ in IR spectra was used for identification of molecular conformation in hydrogel.

Tensile Properties of Hydrogels. The tensile properties of hydrogels were measured in PECF at $25^{\circ} \mathrm{C}$, using a Tensilon UTM-4LH (Orientec Co.) equipped with a $1 \mathrm{~kg}$ load cell. A wrapping film was used at grabbing parts to prevent the hydrogels from breaking or from slippage at the grab. All the hydrogels were tested at a strain rate of $40 \%$ perminute. Mechanical parameters such as Young's modulus $(E)$, tensile strength $\left(\sigma_{\mathrm{B}}\right)$, and strain at break point $\left(\varepsilon_{\mathrm{B}}\right)$ were measured.

Water Vapor Permeability of Hydrogels. Water vapor permeation through the membranes was measured with a cylindrical glass cell ${ }^{10}$ at $37^{\circ} \mathrm{C}$. The rate of water vapor permeability $V_{\mathrm{f}}\left(\mathrm{gm}^{-2}\right.$ day) was determined by weighing the cell at constant time intervals. The exposed membrane area was $12.57 \mathrm{~cm}^{2}$.

Biodegradation of Hydrogels in Vitro. Enzymatic degradation studies in vitro were carried out using papain purchased from Nakarai Chem. Co. Enzyme solution was prepared by standard techniques ${ }^{11}$ at $37^{\circ} \mathrm{C}$. A series of the crosslinked hydrogels was exposed to PECF solution with activator, $0.01 \mathrm{M}$ cystein and $0.04 \mathrm{M}$ EDTA, ${ }^{12}$ at $\mathrm{pH}$ 7.4. Polymer membranes were removed from the 
enzyme solution at appropriate time intervals, weighed and then vacuum dried at $60^{\circ} \mathrm{C}$ to constant weight.

\section{RESULTS AND DISCUSSION}

\section{Relation of Degree of Swelling to Molecular Conformation on Hydrogels}

Linear PHEG, PHPG and PHPeG without crosslinking are water soluble polymers. Molecular conformation of PHEG, PHPG and PHPeG in water are random coil, interrupted $\alpha$-helix (30\% helicity) and complete $\alpha$-helix, respectively. ${ }^{13-15}$ The helix content $X_{\mathrm{H}}$ of linear PHPeEG and PHPePG copolymers in PECF solution at $25^{\circ} \mathrm{C}$ is shown as a function of the mole fraction of HPeG in Figure 1. It is suggested that $\alpha$-helix conformation of PHPeG is disrupted by the introduction of HEG units and is strengthened by HPG units. This can be explained by differences in hydrophobicity of the side chains. The molecular conformation of crosslinked membranes is identified by IR absorption spectra and some spectra in the Amide $\mathrm{V}$ region are shown in Figure 2. The number $X$ in sample code PHPeEG-X denotes the molar percent of $\mathrm{HPeG}$ in copolymer. Although all the membranes showed $\alpha$-helix conformation in xerogel, the absorption of $650 \mathrm{~cm}^{-1}$ which corresponds to random coil conformation increases with decreasing HPeG content in hydrogel. PHPePG-21 hydrogel seems to have higher helix content $X_{\mathbf{H}}$ than PHPeEG-61 hydrogel, which is similar to the result of linear copolymers in Figure 1. Thus, the molecular conformations of linear copolymers in PECF solution are considered to reflect those of crosslinked hydrogels. The results from CD measurement of hydrogels also support this viewpoint. The degree of swelling $Q_{\mathrm{w}}$ of crosslinked membranes in PECF solution is shown in Figure 3. The degree of swelling decreases with increasing $\mathrm{HPeG}$ content in both copolymer membranes. $Q_{\mathrm{w}}$ values of PHPeEG membranes are higher than those of PHPePG membranes,

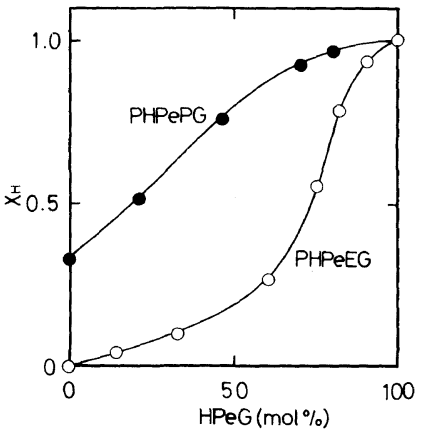

Figure 1. The helix content $X_{\mathrm{H}}$ of linear PHPeEG $(O)$ and PHPePG (O) copolymers as a function of HPeG content $(\mathrm{mol} \%)$ in PECF solution at $25^{\circ} \mathrm{C}$.

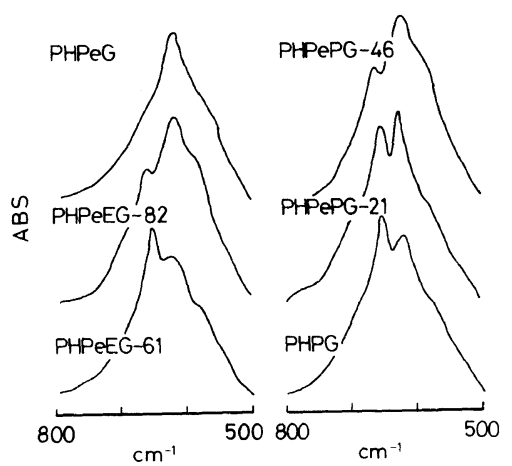

Figure 2. IR absorption spectra in Amide $\mathrm{V}$ region of copolymer membrane in the swollen state.

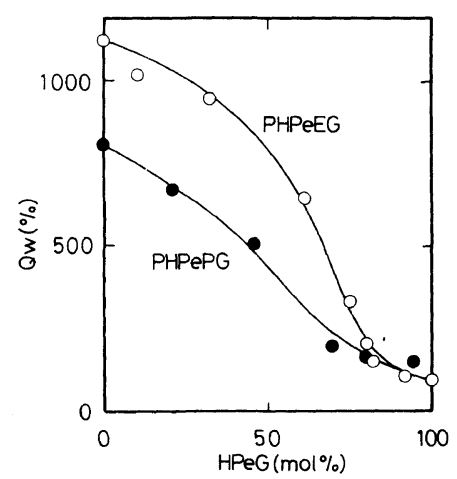

Figure 3. The degree of swelling $Q_{\mathrm{w}}$ of PHPeEG $(O)$ and PHPePG (O) membranes in PECF solution at $25^{\circ} \mathrm{C}$.

which indicates that $Q_{\mathrm{w}}$ also depends on the hydrophobicity of the side chains. However, $Q_{\text {w }}$ of PHPePG-46 membrane is higher than that of PHPeEG-82 membrane which shows 
helix content $X_{\mathrm{H}}$ similar to that of PH-PePG-46 membrane in PECF. This may be due to the less content of hydrophobic HPeG in the PHPePG-46 membrane.

\section{Mechanical Properties of Hydrogels}

The tensile property of human skin shows characteristic behavior with low Young's modulus and high tensile strength. For hydrogels as skin substitutes, it is necessary to have the above characteristic behavior to prevent exfoliation from a wound site. Hydrogels, which can use water as a plasticizer, are considered promising materials as skin substitutes with respect to the tensile property.

The stress-strain (S-S) curves of PHPeEG and of PHPePG hydrogels in PECF are shown in Figure 4 together with the $\mathrm{S}-\mathrm{S}$ curve of human skin at lower abdomen in a longitudinal direction. ${ }^{16}$ The mechanical parameters obtained from $\mathrm{S}-\mathrm{S}$ curves, such as Young's modulus $(E)$, tensile strength $\left(\sigma_{\mathrm{B}}\right)$ and strain at break point $\left(\varepsilon_{\mathrm{B}}\right)$ are listed in Table I. In PHPeEG and PHPePG copolymers $\sigma_{\mathrm{B}}$ and $\varepsilon_{\mathrm{B}}$ increase with increasing molar fraction of HPeG while low Young's modulus is unchanged. Mechanical properties similar to that of human skin are shown in some copolymers. The S-S curves of hydrogels were classified into an elastomer-type having transition point in the low strain region and a skin-type without this point, in our previous paper. ${ }^{4}$ The skin-type behavior was regarded as entropy elasticity without stress relaxation, creeping and hysteresis, while elastomer-type behavior was considered to include the contribution of energy elasticity with stress relaxation, creeping and hysteresis, but without unrecoverable strain. As shown in Figure 4, this transition from skin-type to elastomer-type in $\mathrm{S}-\mathrm{S}$ curve occurs in the region between 82 and 92 mole fraction of $\mathrm{HPeG}$, corresponding to $78-92 \%$ of the helix content $X_{\mathrm{H}}$ and $110-160 \%$ of the degree of swelling $Q_{\mathrm{w}}$ in PHPeE-G copolymers and occurs in the region between 46 and

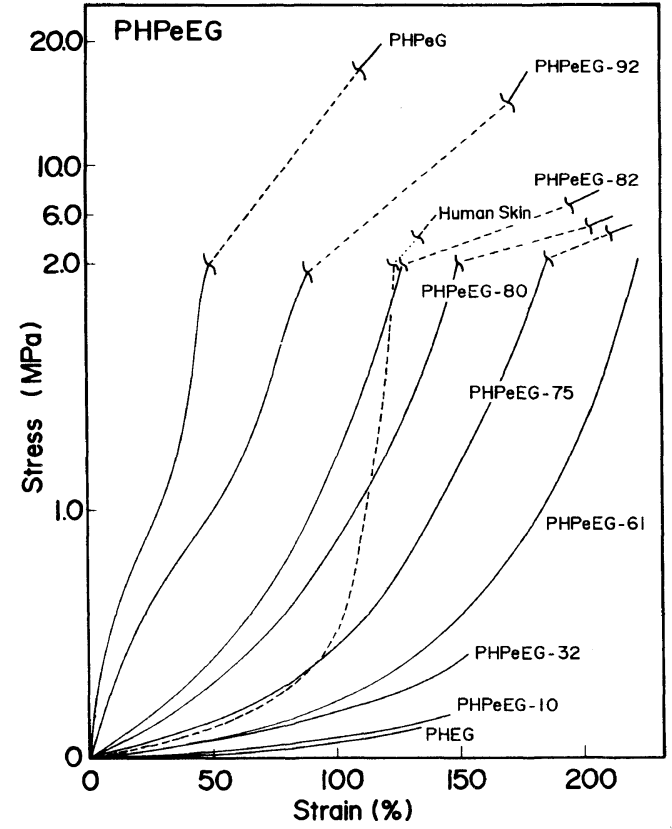

(a)

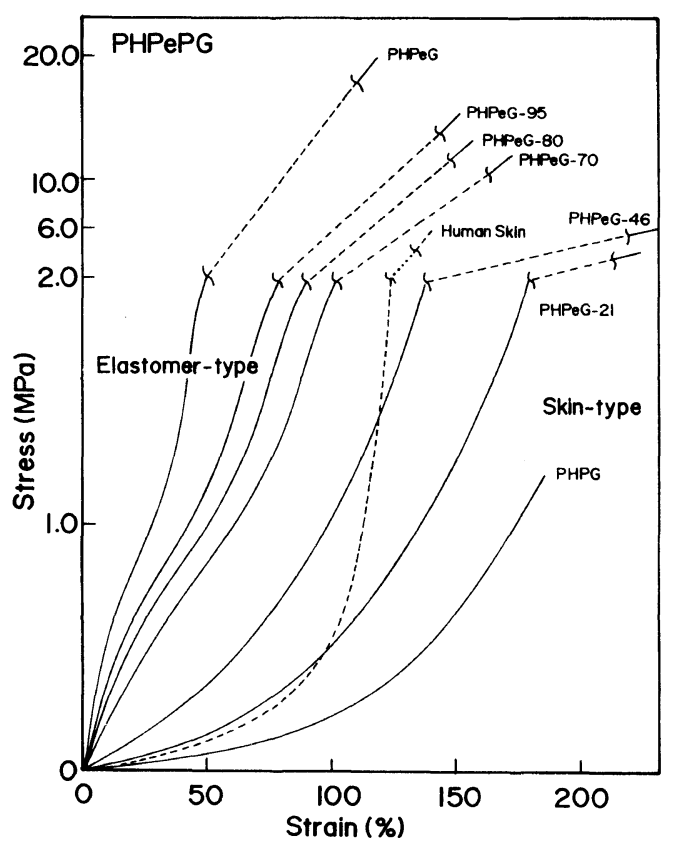

(b)

Figure 4. Stress-strain curves of copolymer membranes, (a) PHPeEG and (b) PHPePG, in PECF solution at $25^{\circ} \mathrm{C}$. 
Table I. Mechanical Parameters obtained from S-S Curves of PHPeEG Membranes in PECF solution at $25^{\circ} \mathrm{C}$

\begin{tabular}{|c|c|c|c|}
\hline \multirow{2}{*}{$\begin{array}{l}\text { Sample } \\
\text { code }\end{array}$} & $E$ & $\sigma_{\mathrm{B}}$ & $\varepsilon_{\mathrm{B}}$ \\
\hline & $\mathrm{MPa}$ & $\mathrm{MPa}$ & $\%$ \\
\hline PHEG & 0.039 & 0.12 & 130 \\
\hline PHPeEG-10 & 0.042 & 0.16 & 150 \\
\hline PHPeEG-32 & 0.045 & 0.45 & 150 \\
\hline PHPeEG-61 & 0.055 & 2.1 & 220 \\
\hline PHPeEG-75 & 0.11 & 3.9 & 220 \\
\hline PHPeEG-80 & 0.17 & 5.8 & 210 \\
\hline PHPeEG-82 & 0.29 & 8.0 & 210 \\
\hline PHPeEG-92 & 6.8 & 18 & 180 \\
\hline PHPeG & 38 & 21 & 120 \\
\hline PHPG & 0.061 & 1.6 & 180 \\
\hline PHPePG-21 & 0.097 & 3.7 & 230 \\
\hline PHPePG-46 & 0.15 & 4.9 & 240 \\
\hline PHPePG-70 & 4.3 & 12 & 200 \\
\hline PHPePG-72 & 5.8 & 12 & 170 \\
\hline PHPePG-80 & 6.9 & 13 & 160 \\
\hline PHPePG-88 & 8.6 & 14 & 160 \\
\hline PHPePG-95 & 12 & 15 & 150 \\
\hline
\end{tabular}

70 mole fraction of $\mathrm{PeG}$, corresponding to $76-95 \%$ of $X_{\mathrm{H}}$ and $200-510 \%$ of $Q_{\mathrm{w}}$ in PHPePG copolymers. It was found that PHPeEG copolymers have the advantage of increasing the mechanical properties while maintaining skin-type behavior compared to PHPePG copolymers. Young's modulus $E$ obtained from initial slope of S-S curves are plotted against the degree of swelling $Q_{\mathrm{w}}$ in Figure 5 and against helix content $X_{\mathbf{H}}$ in Figure 6. In Figure 5, $E$ in PHPePG copolymers is shown to be higher than that of PHPeEG copolymers at the same $Q_{\mathrm{w}}$ owing to higher $X_{\mathrm{H}}$ of PHPePG copolymers. The plot of $E$ against $X_{\mathrm{H}}$ for both copolymers fall on a single curve as can be seen in Figure 6. We proposed in previous paper ${ }^{4}$ that $E$ depends on $X_{\mathrm{H}}$ for PHPeEG copolymers and is related to $X_{\mathrm{H}}$ by

$$
E\left(X_{\mathrm{H}}\right)=\left\{X_{\mathrm{H}} / E_{\mathrm{HPeG}}+\left(1-X_{\mathrm{H}}\right) / E_{\mathrm{HEG}}\right\}^{-1}
$$

where $E_{\mathrm{HPeG}}$ and $E_{\mathrm{HEG}}$ are Young's modulus of PHPeG and PHEG hydrogels, respectively. It was confirmed that $E$, i.e., the shape of S-S curve is governed by $X_{\mathrm{H}}$ in both copolymers.

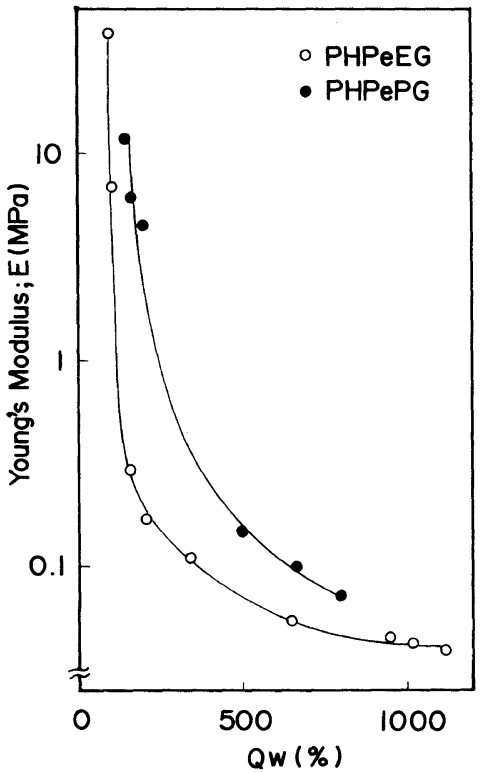

Figure 5. Comparison of PHPeEG copolymers $(\mathrm{O})$ with PHPePG copolymers ( $\boldsymbol{O})$ in the relation between Young's modulus $E$ and degree of swelling $Q_{\mathrm{w}}$ of PECF.

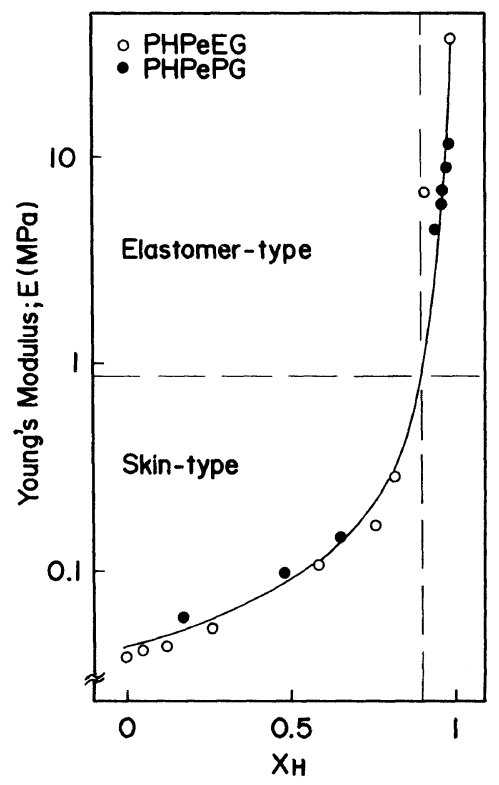

Figure 6. Comparison of PHPeEG copolymers $(\bigcirc)$ with PHPePG copolymers $(\mathbf{)})$ in the relation between Young's modulus $E$ and helix content $X_{\mathbf{H}}$. 
The transition point of S-S curve is considered to correspond to a point $X_{\mathrm{H}}=90 \%$. A small part of random coil residues can provide considerable flexibility to the whole chain segments by interrupting the helix parts.

\section{Water Vapor Permeability of Hydrogels}

A large variety of synthetic polymer membranes has been tested in the treatment of burns. ${ }^{17-19}$ Among them, for example, a crosslinked polymer in the form of hydrogel appears to have added capability for encouraging cellular migration into the graft and vascularization. ${ }^{17}$ In designing a material for effective wound closure or artificial skin inner-layar substitute, at least two functions of skin should be maintained. The first is the ability of skin to keep most bacteria out. The second is its ability to control water passage moderately from tissues and organ. Thus, it is important to know the rate of water vapor permeability $V_{\mathrm{f}}\left(\mathrm{gm}^{-2}\right.$ day) through the membranes. If $V_{\mathrm{f}}$ through the membrane is extremely low, water accumulates at the interface between woundbed and impermeable graft, and edema results. The graft-woundbed interfacial contact is thereby undermined. Consequently, a skin substitute membrane should have higher $V_{\mathrm{f}}$, than that of the human physiological level of about $V_{\mathrm{f}}=500\left(\mathrm{gm}^{-2}\right.$ day $)^{20}$ in order to maintain the ability to wet the woundbed and maintain an air-free interface. Figure 7 shows the relation between water vapor permeability $V_{\mathrm{f}}\left(\mathrm{g} \mathrm{m}^{-2}\right.$ day $)$ of PECF and degree of swelling $Q_{\mathrm{w}}$ for PHPeEG and PHPePG copolymer membranes at $37^{\circ} \mathrm{C}$. It is shown that $V_{\mathrm{f}}$ is highly dependent on the $Q_{\mathrm{w}}$ in PECF and meets the requirement of being a skin substitute; i.e., $V_{\mathrm{f}}$ is higher than human physiological level in all the copolymers. When $V_{\mathrm{f}}$ of PHPeEG and PHPePG membranes are compared at the same $Q_{\mathrm{w}}, V_{\mathrm{f}}$ of PHPeEG is higher than that of PHPePG membranes, indicating that the molecular conformation affects the state of water in membranes.

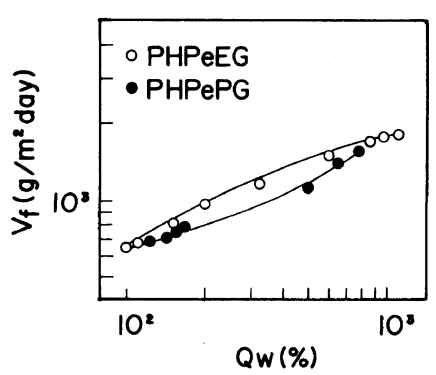

Figure 7. Comparison of PHPeEG copolymers $(O)$ with PHPePG copolymers $(\mathcal{O})$ in the relation between the rate of water vapor permeability $V_{\mathrm{f}}\left(\mathrm{g} / \mathrm{m}^{2}\right.$ day) and degree of $Q_{w}$ of PECF at $37^{\circ} \mathrm{C}$.

\section{Biodegradation of Hydrogels in Vitro}

Numerous proteases may be present at a wound site. ${ }^{21}$ These proteases are divided into some classes depending on the structure of active site. Enzymes of inflammatory response that are likely to degrade poly $(\alpha$-amino acid $) s$ include the endopeptidase Cathepsin B, exopeptidases Carboxypeptidase and Leucine amino peptidase. ${ }^{22}$ In the present investigation, the plant thiol endopeptidase Papain was selected as a commercially available analogue of Cathepsin B. Papain is an ellipsoidal protein molecule with approximate dimensions $5.0 \times$ $3.7 \times 3.7 \mathrm{~nm}^{23}$ Cathepsin $\mathrm{B}$ is a thiol endopeptidase isolated from mammalian spleen, liver, kidney and lung, and released by the cells of inflated to cathepsin B. Although Papain is a general thiol endopeptidase, it has preference for peptide bonds where the amino acid residue of the carbonyl group is arginine, lysine or glutamine and where this amino acid is joined on either side by amino acids with hydrophobic side chains. ${ }^{24}$ Pre-weighed crosslinked copolymer membranes were exposed to $0.1 \mathrm{mg}$ of Papain in $1 \mathrm{ml}$ of PECF with the activator at $37^{\circ} \mathrm{C}$ and $\mathrm{pH} 7.4$, and the results obtained with PHPG membrane are illustrated in Figure 8. Although Papain is a macromolecule whose molecular weight is about 21,000 , it is apparent that the polymer network does not exclude the Papain molecule. Degradation of PHPG was also measured by changes in the degree of 


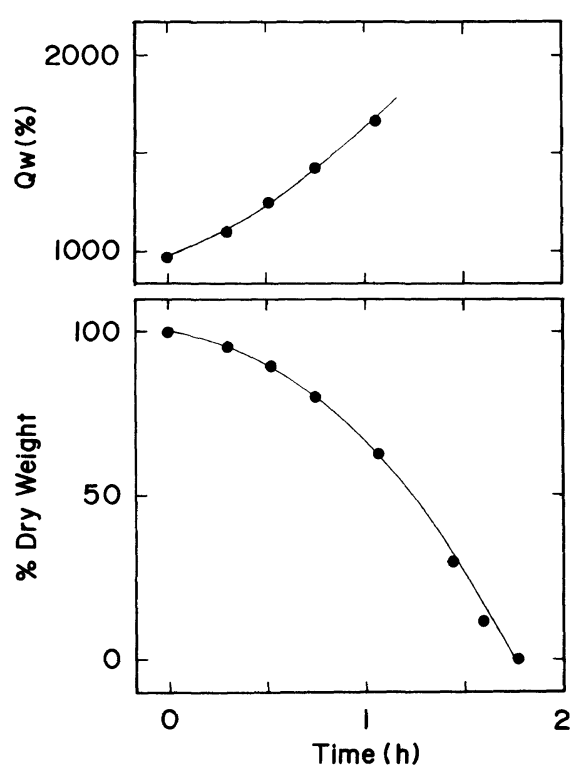

Figure 8. Dry weight ratio (\%) and the degree of swelling $Q_{\mathrm{w}}$ of PECF for PHPG membrane as a function of papain digestion time (h) at $37^{\circ} \mathrm{C}$ and $\mathrm{pH}=7.4$.

swelling $Q_{\mathrm{w}}$. Since an immediate increase in $Q_{\mathrm{w}}$ was observed, the degradation must be a bulk rather than a surface phenomenon. Geometrical considerations are also consistent with easy access of the enzyme to the bulk of the membrane. As shown in Figure 8, with Papain digestion an immediate increase in $Q_{\mathrm{w}}$ of PHPG membrane was observed, while weight loss occurred a little bit more slowly. An endopeptidase must make two incisions in a chain segment to produce a soluble fragment, but a single cleavage will decrease the effective crosslink density, resulting in increased $Q_{\mathrm{w}}$ of membranes. Thus, the initial effect of Papain is to decrease the effective crosslink density without producing soluble materials. Figure 9 summarizes the rate of Papain digestion $V_{1 / 2}$ $\left(\mathrm{h}^{-1}\right)$ as a function of $Q_{\mathrm{w}}$ in PECF for PHPeEG and PHPePG copolymers. $V_{1 / 2}$ is defined as the reciprocal of time required for sample weight to be reduced to one-half its initial value. It is also clearly shown that a common relation the same as in the previous paper, ${ }^{25}$ is obtained between $V_{1 / 2}$ and $Q_{\mathrm{w}}$ in both copolymers. The

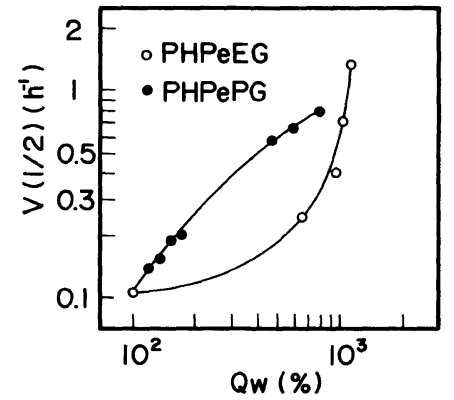

Figure 9. Comparison of PHPeEG copolymers $(O)$ with PHPePG copolymers $(O)$ in the relation between the rate of papain digestion $V_{1 / 2}\left(\mathrm{~h}^{-1}\right)$ and degree of swelling $Q_{\mathrm{w}}$ of $\mathrm{PECF}$ at $37^{\circ} \mathrm{C}$ and $\mathrm{pH}=7.4$.

order of the rate of Papain digestion in two copolymers was PHPePG $>$ PHPeEG at the same $Q_{\mathrm{w}}$. Hydrophobic effects of the helix region are considered to be important in explaining the faster digestion of higher helix content $X_{\mathrm{H}}$ in comparing two copolymers.

The major conclusions are as follows.

(1) PHPeEG and PHPePG copolymers are considered to be promising materials as skin substitutes, showing high degree of swelling, skin-type tensile behavior, high water vapor permeability and enzymatic degradation behavior in vitro.

(2) PHPePG copolymers show higher helix content $X_{\mathbf{H}}$ than PHPeEG copolymers at the same degree of swelling $Q_{\mathrm{w}}$, due to different hydrophobicities.

(3) Young's modulus $E$ and tensile behavior are shown to be governed by molecular conformation of copolymers.

(4) PHPeEG copolymers show higher water vapor permeability and slower enzymatic digestion than PHPePG copolymers at the same $Q_{w}$, which is considered due to differences in helix content.

\section{REFERENCES}

1. S. D. Bruck, Biomater. Med. Dev. Artif. Organs, 1, 79 (1973).

2. B. D. Ratner and A. S. Hoffman, in "Hydrogels for 
Medical and Related Applications," J. D. Andrade, Ed., ACS Symp. No. 31, ACS, Washington D. C., 1976, Chapter 1.

3. J. M. Anderson, K. L. Spilizewski, and A. Hiltner, in "Biocompatibility of Tissue Analogs," D. F. Williams, Ed., CRC Press, Boca Raton, FL, 1985, p 68.

4. E. Nakanishi, E. Sugiyama, Y. Shimizu, S. Hibi, and T. Hayashi, Polym. J., 23, 983 (1991).

5. C. A. Homsey, J. Biomed. Mater. Res., 4, 341 (1971).

6. T. Hayashi, E. Nakanishi, and A. Nakajima, Kobunshi Ronbunshu, 43, 633 (1986).

7. H. E. Auer and P. Doty, Biochemistry, 5, 1708 (1966).

8. T. Sugie and P. A. Hiltner, J. Macromol. Sci. -Phys., B17; 769 (1980).

9. T. Miyazawa, J. Masuda, and K. Fukushima, $J$. Polym. Sci., 62, 62, (1966).

10. T. Hayashi, K. Takeshima, E. Kobatake, and A. Nakajima, Kobunshi Ronbunshu, 42, 777 (1985).

11. H. B. Bensusan, Biochemistry, 8, 4716 (1969).

12. J. Lowbridge and J. S. Fruton, J. Biol. Chem., 249, 6754 (1974).

13. N. Lotan, A. Yaron, A. Berger, and M. Sela, Biopolymers, 3, 625 (1965).
14. N. Lotan, A. S. Yaron, and A. Berger, Biopolymers, 4, 365 (1966).

15. N. Lotan, N. Bixon, and A. Berger, Biopolymers, 8, 247 (1969).

16. H. Yamada, in "Strength of Biological Materials," F. G. Evans, Ed., WWC Press, 1970, p 226.

17. J. Hubacek, K. Kliment, J. Dusek, and J. Hubacek, J. Biomed. Mater. Res., 1, 387 (1967).

18. I. R. Schmolka, J. Biomed. Mater. Res., 6, 571 (1972).

19. J. Guldalian, C. Jelenko, III, D. Calloway, and J. T. McKnight, J. Trauma., 13, 32 (1973).

20. I. V. Yannas and J. F. Burke, J. Biomed. Mater. Res., 14, 65 (1980).

21. D. F. Williams, J. Bioeng., 1, 279 (1977).

22. T. N. Salthouse, J. Biomed. Mater. Res., 10, 197 (1976).

23. J. Drenth, J. N. Jansonius, R. Koekoek, and B. G. Solthers, "Papain, X-Ray Structure, in the Enzymes," Vol. III, P. D. Boyer, Ed., Academic Press, New York, N. Y., 1971, p 485.

24. J. Lowbridge and J. S. Fruton, J. Biol. Chem., 249, 6754 (1974).

25. T. Hayashi, E. Nakanishi, and A. Nakajima, Polym. J., 19, 1025 (1987). 